\title{
Quantum radiation in external background fields
}

\author{
Ralf Schützhold, Günter Plunien and Gerhard Soff \\ Institut für Theoretische Physik, Technische Universität Dresden, \\ Mommsenstr. 13, D-01062 Dresden, Federal Republic of Germany
}

\begin{abstract}
A canonical formalism is presented which allows for investigations of quantum radiation induced by localized, smooth disturbances of classical background fields by means of a perturbation theory approach. For massless, non-selfinteracting quantum fields at zero temperature we demonstrate that the low-energy part of the spectrum of created particles exhibits a non-thermal character. Applied to QED in varying dielectrics the response theory approach facilitates to study two distinct processes contributing to the production of photons: the squeezing effect due to space-time varying properties of the medium and of the velocity effect due to its motion. The generalization of this approach to finite temperatures as well as the relation to sonoluminescence is indicated.
\end{abstract}

PACS-numbers: 12.20.; 42.50.; 04.60.Ds; 78.60.Mq

Keywords:

Dynamical Casimir effect; QED in varying dielectrics; Sonoluminescense;

\section{Introduction}

Perhaps one of the most fascinating aspects of the static Casimir effect [1, 2] is that it demonstrates the highly non-trivial nature of the vacuum state in quantum field theory (see Refs. [3, 4, 5] for reviews). During the last decade the dynamical Casimir effect, i.e. the production of particles due to the presence of space-time varying external conditions has become subject of an increasing number of studies especially devoted to particle creation in gravitational backgrounds (see for a review [5, 6), due to dynamical boundary conditions (see Refs. in [7]) and photon production in moving dielectrics (see e.g. [8, 9, 10]) to mention only a very few of prominent fields of research.

The phenomenon of quantum radiation $(\mathrm{QR})$ can be interpreted as the response of the vacuum of quantized fields due to their interactions with external conditions. One may think of 
the latter as some space-time dependent background fields (classical field configuration) or as time-dependent, geometrical boundary conditions superimposed on the quantized fields under consideration (e.g. moving mirrors or dielectrics).

In a recent investigation [7] we propose a canonical approach to the dynamical Casimir effect in the presence of boundary conditions which facilitates to calculate the number of generated particles by means of response theory. In this article we are going to apply this approach to the situation of quantum fields in arbitrary space-time dependent background fields lying particular emphasis on the situation of QED in moving and space-time varying dielectrics. Under rather general conditions the low-energy behaviour of the spectrum of produced particles can be deduced. We also indicate that the formalism is most suitable for incorporating finite-temperature effects as well. This paper is organized as follows: In section 2 we deduce some features of the energy spectrum of the dynamical Casimir effect in smooth background fields based on the response theory approach. In section 3 the formalism is applied to QED in dynamical dielectrics lying particular emphasis on the problem of field quantization. An interaction Hamiltonian is derived which describes particle production due to two distinct generic prossess: 1. squeezing of the QED ground state due to localized, space-time varying dielectric properties inside the medium; 2. velocity effect due to motion of the medium. Section 4 examines the squeezing effect where a general expression for the total radiated energy will be derived. Section 5 discusses the velocity effect qualitatively to underline the importance of realistic ansätze for the velocity field and how they may affect the energy-spectrum of the produced particles. We will close with some discussions devoted to the following issues: generalization of the formalism to finite temperatures, application to media with nontrivial permeability function and an indication of how to estimate the contribution of QR to the phenomenon of sonoluminescence.

\section{Energy spectrum of quantum radiation}

In this section we present the general formalism for the calculation of the number of particles produced by the dynamical Casimir effect. We consider a set of quantized fields $\hat{\Theta}_{A}$ interacting with a classical background described by some smooth, space-time dependent functions $\Delta K_{\mu \nu}^{A B}$. The rather general system under consideration may be characterized by the four suppositions:

$I \quad$ : The $\hat{\Theta}_{A}$ are supposed to be massless and non-selfinteracting bosonic fields .

II : The field configuration is considered at zero temperature and initially in the vacuum state.

III : A proper definition of particles is provided by the unperturbed Hamiltonian $\hat{H}_{0}$.

$I V$ : The interaction Hamiltonian $\hat{H}_{1}$ is determined by small, space-time localized and smooth functions $\Delta K_{\mu \nu}^{A B}$.

These four assumptions allow to deduce the low-energy behaviour of the spectrum $e(\omega)$ of the 
produced particles. We shall prove that for small freqencies $\omega$ the energy spectrum $e(\omega)$ will be proportional to $\omega^{4}$ in contrast to the thermal spectrum which behaves as $\omega^{3}$.

At first we specify a class of Lagrangians compatible with the four assumptions stated above. Due to supposition $I$ we assume $\hat{\mathcal{L}}$ as a bilinearform of first order derivatives of the fields $\hat{\Theta}_{A}$ interacting with background fields $\Delta K_{\mu \nu}^{A B}$ :

$$
\hat{\mathcal{L}}=\frac{1}{2} \partial^{\mu} \hat{\Theta}_{A}\left(K_{\mu \nu}^{A B}+\Delta K_{\mu \nu}^{A B}(\vec{r}, t)\right) \partial^{\nu} \hat{\Theta}_{B}
$$

with $\Delta K_{\mu \nu}^{A B}(\vec{r}, t) \rightarrow 0$ for $|\vec{r}| \rightarrow \infty$ and for $t \rightarrow \pm \infty$ (according to IV). In Eq. (1) and in the following formulae we make use of the summation convention and declare that one has to sum over all indices $(A, \mu, i$ etc.) that do not occour at both sides of the equation.

This leads to the total Hamiltonian :

$$
\hat{H}(t)=\hat{H}_{0}+\hat{H}_{1}(t)=\int d^{3} r\left(\hat{\mathcal{H}}_{0}+\hat{\mathcal{H}}_{1}(t)\right)
$$

with $\hat{\mathcal{H}}_{1}(\vec{r}, t) \rightarrow 0$ for $|\vec{r}| \rightarrow \infty$ and for $t \rightarrow \pm \infty$ (according to $I V$ ) together with the undisturbed Hamiltonian density

$$
\hat{\mathcal{H}}_{0}=\frac{1}{2} \hat{\Pi}_{A} T^{A B} \hat{\Pi}_{B}+\frac{1}{2} V_{i j}^{A B} \partial^{i} \hat{\Theta}_{A} \partial^{j} \hat{\Theta}_{B} \quad,
$$

assuming the constants $K_{\mu \nu}^{A B}$ satisfy (possibly after a suitable transformation) $K_{0 i}^{A B}=K_{i 0}^{A B}=0$ or other appropriate conditions leading to the constant matrices $T^{A B}$ and $V_{i j}^{A B}$. A proper definition of particles with respect to the undisturbed Hamiltonian $\hat{H}_{0}$ (according to III) is provided by insertion of the expansions

$$
\hat{\Theta}_{A}(\vec{r}, t)=V^{-1 / 2} F_{A}^{B} \underset{\vec{k}}{£} \sqrt{\frac{1}{2 \omega_{\vec{k} B}}}\left(\hat{a}_{\vec{k} B}^{+}(t) e^{i \vec{k} \vec{r}}+\text { h.c. }\right)
$$

for the fields and

$$
\hat{\Pi}_{A}(\vec{r}, t)=V^{-1 / 2} G_{A}^{B} \underset{\vec{k}}{£} i \sqrt{\frac{\omega_{\vec{k} B}}{2}}\left(\hat{a}_{\vec{k} B}^{+}(t) e^{i \vec{k} \vec{r}}-\text { h.c. }\right)
$$

for the canonical field momenta. ( $V$ denotes the quantization volume.) 
Supposition III demands the existence of appropriate matrices $F_{B}^{A}$ and $G_{B}^{A}$ which allow to diagonalize $\hat{H}_{0}$ (i.e. terms like $\hat{a}_{\vec{k} A} \hat{a}_{\vec{k}^{\prime} B}$ and $\hat{a}_{\vec{k} A}^{+} \hat{a}_{\vec{k}^{\prime} B}^{+}$generated by the insertion of (4) and (5) into (3) have to cancel) leading to the form

$$
\hat{H}_{0}=\oiint_{\vec{k} A} \omega_{\vec{k} A}\left(\hat{a}_{\vec{k} A}^{+} \hat{a}_{\vec{k} A}+\frac{1}{2}\right)=\oiint_{\vec{k} A} \omega_{\vec{k} A}\left(\hat{N}_{\vec{k} A}+\frac{1}{2}\right) .
$$

The $\hat{H}_{0}$-frequencies $\omega_{\vec{k} A}$ possess a quasi-linear dispersion relation (after $I$ ) :

$$
\omega_{\vec{k} A}=\sqrt{k_{i} k_{j} W_{A}^{i j}}=\mathcal{O}(k=|\vec{k}|) .
$$

The following calculations are most suitably performed in interaction-representation:

$$
\begin{aligned}
& \frac{d \hat{A}}{d t}=i\left[\hat{H}_{0}, \hat{A}\right]+\frac{\partial \hat{A}}{\partial t}, \\
& \frac{d}{d t}|\psi\rangle=-i \hat{H}_{1}(t)|\psi\rangle .
\end{aligned}
$$

Suppositions II and III imply the following definition of the vacuum - the initial state - as well as that of proper particle creation/annihilation operators $\hat{a}_{\vec{k} A}^{+} / \hat{a}_{\vec{k} A}$ :

$$
|\psi(t \rightarrow-\infty)\rangle=|0\rangle \quad \text { with } \quad \forall \vec{k} A \quad \hat{a}_{\vec{k} A}|0\rangle=0 \quad .
$$

Eq. (9) can be formally integrated with the time-ordering operator $\mathcal{T}$ :

$$
\begin{aligned}
|\psi(t \rightarrow \infty)\rangle & =\mathcal{T}\left[\exp \left(-i \int d t \hat{H}_{1}(t)\right)\right]|0\rangle \\
& =\mathcal{T}\left[\exp \left(-i \int d^{4} x \hat{\mathcal{H}}_{1}(\underline{x})\right)\right]|0\rangle \\
& =\sum_{n=0}^{\infty} \frac{(-i)^{n}}{n !} \int d t_{n} \cdots \int d t_{1} \mathcal{T}\left[\hat{H}_{1}\left(t_{n}\right) \cdots \hat{H}_{1}\left(t_{1}\right)\right]|0\rangle \\
& =\sum_{n=0}^{\infty} \frac{(-i)^{n}}{n !} \int d^{4} x_{n} \cdots \int d^{4} x_{1} \mathcal{T}\left[\hat{\mathcal{H}}_{1}\left(\underline{x}_{n}\right) \cdots \hat{\mathcal{H}}_{1}\left(\underline{x}_{1}\right)\right]|0\rangle,
\end{aligned}
$$

with $\underline{x}=(\vec{r}, t)$. The expectation value of the number opertator is given by

$$
\begin{aligned}
\left\langle\hat{N}_{\vec{k} A}\right\rangle= & \left\langle\psi(t \rightarrow \infty)\left|\hat{N}_{\vec{k} A}\right| \psi(t \rightarrow \infty)\right\rangle \\
= & \sum_{m=1}^{\infty} \frac{(+i)^{m}}{m !} \int d^{4} x_{m} \cdots \int d^{4} x_{1} \sum_{n=1}^{\infty} \frac{(-i)^{n}}{n !} \int d^{4} x_{n}^{\prime} \cdots \int d^{4} x_{1}^{\prime} \\
& \left\langle 0\left|\mathcal{T}\left[\hat{\mathcal{H}}_{1}\left(\underline{x}_{m}\right) \cdots \hat{\mathcal{H}}_{1}\left(\underline{x}_{1}\right)\right] \hat{N}_{\vec{k} A} \mathcal{T}\left[\hat{\mathcal{H}}_{1}\left(\underline{x}_{n}^{\prime}\right) \cdots \hat{\mathcal{H}}_{1}\left(\underline{x}_{1}^{\prime}\right)\right]\right| 0\right\rangle .
\end{aligned}
$$


The vacuum expectation values have to be calculated in the $\hat{H}_{0}$-dynamics and can also be deduced from the $2(n+m+1)$-point correlation functions $\left\langle 0\left|\hat{\Theta}_{A_{1}}\left(\underline{x}_{1}\right) \cdots \hat{\Theta}_{A_{p}}\left(\underline{x}_{p}\right)\right| 0\right\rangle$. Due to assumption $I$ the pertubation Hamiltonian density can be expressed as:

$$
\hat{\mathcal{H}}_{1}=Q_{\mu \nu}^{A B}(\underline{x}) \partial^{\mu} \hat{\Theta}_{A} \partial^{\nu} \hat{\Theta}_{B}
$$

In the interaction representation the time evolution of the fields is now governed by $\hat{H}_{0}$ and assumes the form:

$$
\hat{\Theta}_{A}(\underline{x})=V^{-1 / 2} F_{A}^{B} \underset{\vec{k}}{£} \sqrt{\frac{1}{2 \omega_{\vec{k} B}}}\left(\hat{a}_{\vec{k} B}^{+} e^{i \underline{k}_{B} \underline{x}}+\text { h.c. }\right)
$$

with $\underline{k}_{A}=\left(\vec{k}, \omega_{\vec{k} A}\right)$. The interaction term becomes

$$
\begin{aligned}
\int d^{4} x \hat{\mathcal{H}}_{1}(\underline{x})= & \underset{\vec{k}_{1} \vec{k}_{2}}{£} \frac{k_{1 C}^{\mu} k_{2 D}^{\nu}}{2 V \sqrt{\omega_{\vec{k}_{1} C^{\omega_{k_{2} D}}}}} \times \\
& \int d^{4} x Q_{\mu \nu}^{A B}(\underline{x}) F_{A}^{C} F_{B}^{D}\left(\hat{a}_{\vec{k}_{1} C}^{+} e^{i \underline{k}_{1} \underline{x}}+\text { h.c. }\right)\left(\hat{a}_{\vec{k}_{2} D}^{+} e^{i \underline{k}_{2} \underline{x}}+\text { h.c. }\right)
\end{aligned}
$$

In order to obtain a non-zero matrix-element in (12) at the left and at the right of $\hat{N}_{\vec{k} A}$ at least one summation index $\vec{k}_{\ell}$ has to be equal to $\vec{k}$, which generates an overall factor of order $k$. Assuming the functions $Q_{\mu \nu}^{A B}(\underline{x})$ to be localized and smooth enough $(I V)$, all mode summations are convergent and the $d^{4} x$-integrals are finite for the limit $k \rightarrow 0$. Under these conditions, we can conclude that the number of particles per mode possess the low-momentum behaviour

$$
\left\langle\hat{N}_{\vec{k} A}\right\rangle=\mathcal{O}(k)
$$

This enables us to calculate the total radiated energy associated with the total number of created particles:

$$
E=\oiint_{\vec{k} A} \omega_{\vec{k} A}\left\langle\hat{N}_{\vec{k} A}\right\rangle \rightarrow \frac{V}{(2 \pi)^{3}} \sum_{A} \int d^{3} k \omega_{\vec{k} A}\left\langle\hat{N}_{\vec{k} A}\right\rangle=\sum_{A} \int_{0}^{\infty} d \omega_{A} e_{A}\left(\omega_{A}\right)=\int_{0}^{\infty} d \omega e(\omega)
$$

In the limiting case of small frequencies $\omega$ simple power counting leads to a non-thermal spectral energy density:

$$
e(\omega) \sim \omega^{4}+\mathcal{O}\left(\omega^{5}\right)
$$

This reveals a general feature of the low-energy part of the spectrum of particles created by the dynamical Casimir effect due to the interaction with external background fields, assuming that the suppositions $I-I V$, which describe a very general case, are fulfilled. 


\section{QED in dielectrics}

\subsection{Equations of motion}

Throughout this article natural units with $\hbar=c=\varepsilon_{0}=\mu_{0}=1$ will be used. We start with the source free Maxwell equations (i.e. for $\rho=0$ and $\vec{j}=\overrightarrow{0}$ ) :

$$
\begin{array}{ll}
\nabla \vec{B}=0, & \nabla \times \vec{E}=-\dot{\vec{B}} \\
\nabla \vec{D}=0 \quad, & \nabla \times \vec{H}=\dot{\vec{D}} .
\end{array}
$$

The first pair (19) leads to the definition of the potentials :

$$
\vec{B}=-\nabla \times \vec{A} \quad, \quad \vec{E}=\dot{\vec{A}}+\nabla \Phi
$$

Now we suppose a linear and nondispersive medium in arbitrary motion described by the scalar dielectricity function $\varepsilon(\vec{r}, t)$ and a velocity field $\vec{\beta}(\vec{r}, t)$. For the special case of a medium globally at rest, i.e. $u^{\mu}=(1, \vec{\beta}=\overrightarrow{0})$, where the relations $\vec{D}=\varepsilon \vec{E}$ and $\vec{H}=\vec{B}$ hold, the Lagrangian leading to the second pair of the Maxwell equations reads:

$$
\mathcal{L}=\frac{1}{2}\left(\varepsilon \vec{E}^{2}-\vec{B}^{2}\right)
$$

There are different options for the construction of the Lagrangian for the general case of arbitary $u^{\mu}(\vec{r}, t)$ and $\varepsilon(\vec{r}, t)$. The most direct way is to make an ansatz as a linear combination of all possible Lorentz-covariant scalars constructed from the tensors at hand: the field strength tensor $F^{\mu \nu}$, the Lorentz metric $g_{\mu \nu}$, the four velocity $u_{\mu}$, possibly the Levi-Civita pseudo-tensor $\epsilon_{\mu \nu \rho \sigma}$ and the dielectricity function $\varepsilon$ (Lorentz-scalar). For reasons of simplicity one may neglect additional acceleration effects that could be carried by $u_{\mu, \nu}$ or $\varepsilon_{, \mu}$ etc. The only form that reduces to the special case (22) of a medium at rest and the vacuum Lagrangian $\mathcal{L}=\frac{1}{4} F_{\mu \nu} F^{\nu \mu}$ for $\varepsilon=1$, is provided by

$$
\begin{aligned}
\mathcal{L} & =\frac{1}{4} F_{\mu \nu} F^{\nu \mu}+\frac{\varepsilon-1}{2} u^{\mu} F_{\mu \nu} F^{\nu \rho} u_{\rho} \\
& =\frac{1}{2}\left(\vec{E}^{2}-\vec{B}^{2}\right)-\frac{\varepsilon-1}{2}\left(1-\vec{\beta}^{2}\right)^{-1}\left((\vec{\beta} \vec{E})^{2}-(\vec{E}-\vec{\beta} \times \vec{B})^{2}\right)
\end{aligned}
$$

This Lagrangian established by means of covariance arguments may be expanded in powers in $\vec{\beta}$ (assuming $|\vec{\beta}| \ll 1$ ) resulting in the non-covariant form

$$
\mathcal{L}=\frac{1}{2}\left(\varepsilon \vec{E}^{2}-\vec{B}^{2}\right)+(\varepsilon-1) \vec{\beta}(\vec{E} \times \vec{B})+\mathcal{O}\left(\vec{\beta}^{2}\right)
$$


The Euler-Lagrange equations for the scalar potential $\Phi$ lead to:

$$
\nabla \frac{\partial \mathcal{L}}{\partial \nabla \Phi}=\nabla \vec{D}=0
$$

while the equations of motion for $\vec{A}$ read:

$$
\frac{\partial}{\partial t} \frac{\partial \mathcal{L}}{\partial \dot{A}^{i}}=\dot{D}_{i}=-\frac{\partial}{\partial x^{j}} \frac{\partial \mathcal{L}}{\partial A_{, j}^{i}}=(\nabla \times H)_{i}
$$

So the Euler-Lagrange equations are identical with the second pair of the Maxwell equations, if we introduce the electric and magnetic displacement fields $\vec{D}$ and $\vec{H}$ according to

$$
\vec{D}=\frac{\partial \mathcal{L}}{\partial \vec{E}}=\varepsilon \vec{E}+(\varepsilon-1) \vec{B} \times \vec{\beta}+\mathcal{O}\left(\vec{\beta}^{2}\right)
$$

and

$$
\vec{H}=-\frac{\partial \mathcal{L}}{\partial \vec{B}}=\vec{B}+(\varepsilon-1) \vec{E} \times \vec{\beta}+\mathcal{O}\left(\vec{\beta}^{2}\right)
$$

Applying Euler's theorem the Lagrangian (23) transforms into the alternative form

$$
\mathcal{L}=\frac{1}{2}(\vec{E} \vec{D}-\vec{B} \vec{H})
$$

which holds to all orders in $\vec{\beta}$.

\subsection{Gauge problems}

If we try to carry out the canonical quantization based on the Hamiltonian formalism applied to the Lagrangian obtained above, we are faced with some problems arising from the fact, that QED is a gauge field theory. (Attempts for quantizing QED in dielectric media date back for more than half of a century [11, 12]. To solve the quantization problem for general media has attracted recent interest in quantum optics, see [13]-[20].) Due to the fact, that $\mathcal{L}$ contains no kinetic term for the scalar field component $\Phi$ there exists the primary constraint:

$$
\Pi_{0}=\frac{\partial \mathcal{L}}{\partial \dot{\Phi}}=0 .
$$

A secondary constraint arises from the absence of any potential term for the longitudinal component of the vector potential $\vec{A}_{\|}$:

$$
\nabla \vec{\Pi}=\nabla \frac{\partial \mathcal{L}}{\partial \dot{\vec{A}}}=\nabla \frac{\partial \mathcal{L}}{\partial \vec{E}}=\nabla \vec{D}=0
$$

So far these problems also occur in vacuum QED. However, in that case it is possible to remove both kinds of field modes simultaneously by choosing the Coulomb gauge $\nabla \vec{A}=0$ which has 
$\Phi=0$ as immediate consequence. Equivalently, one can choose the temporal gauge $\Phi=0$ which directly leads to $\nabla \vec{A}=0$. For a detailed discussion of the problem of the quantization with constraints we refer e.g. to [21]. So in vacuum QED it is possible to remove the scalar and the longitudinal photons simultaneously. This fails however for QED in media because of the non-trivial relation between $\vec{E}$ and $\vec{D}$. As the next step we observe that the Hamiltonian derived according to the canonical prescription

$$
\mathcal{H}(\vec{\Pi}, \vec{A}, \Phi)=\vec{\Pi} \dot{\vec{A}}-\mathcal{L}=\frac{1}{2}(\vec{E} \vec{D}+\vec{B} \vec{H})-\vec{\Pi} \nabla \Phi
$$

contains the term $\vec{\Pi} \nabla \Phi$. Even though $\nabla \vec{\Pi}=0$, dropping this term or setting $\Phi=0$ would change the equations of motion, e.g. such that they are no more consistent with the Coulomb gauge $\nabla \vec{A}=0$. One way to solve the gauge problem is provided by carrying out the procedure of Dirac quantization as described e.g. in [21. One could also try to generalize the path integral quantization recently applied to dielectrics at rest 20]. Here we proceed in a different way: Based on a decomposition of the electromagnetic fields into an appropriatly choosen basis $\left\{\vec{f}_{a}\right\}$ we introduce a set of independent variables $\left\{q_{a}\right\}$ leading to a Lagrangian $L(q, \dot{q}, t)$ without any constraints and perform the usual canonical quantization. It will be necessary to prove that the primary and secondary constraints required for the fields can be satisfied by the adequate choice for $\left\{\overrightarrow{f_{a}}\right\}$.

For this purpose we fix the gauge by choosing the Coulomb condition:

$$
\nabla \vec{A}=0
$$

As mentioned above, in dielectrics this does not automatically imply $\Phi=0$. Furthermore, from the equation of motion $\nabla \vec{D}=0$ it follows:

$$
\nabla(\varepsilon \nabla \Phi)=-\nabla(\varepsilon \dot{\vec{A}}+(\varepsilon-1) \vec{\beta} \times(\nabla \times \vec{A}))+\mathcal{O}\left(\vec{\beta}^{2}\right)
$$

which possesses non-trivial solutions $\Phi \neq 0$. Requiring the boundary conditions $\Phi \rightarrow 0$ for $|\vec{r}| \rightarrow \infty$ Eq. (34) is solvable with the aid of the Green function $G\left(\vec{r}, \vec{r}^{\prime}, t\right)$ corresponding to the Laplace-Beltrami-type operator $(\nabla \varepsilon \nabla)$ :

$$
\begin{aligned}
\Phi(\vec{r}, t)= & -\int d^{3} r^{\prime} G\left(\vec{r}, \vec{r}^{\prime}, t\right) \times \\
& \nabla^{\prime}\left(\varepsilon\left(\vec{r}^{\prime}, t\right) \dot{\vec{A}}\left(\vec{r}^{\prime}, t\right)+\left(\varepsilon\left(\vec{r}^{\prime}, t\right)-1\right) \vec{\beta}\left(\vec{r}^{\prime}, t\right) \times\left(\nabla^{\prime} \times \vec{A}\left(\vec{r}^{\prime}, t\right)\right)\right)+\mathcal{O}\left(\vec{\beta}^{2}\right) .
\end{aligned}
$$

This equation explicitly reveals the nature of the scalar potential $\Phi$ as a dependent field variable. Since Eq. (34) represents an equation of motion the action $\mathcal{A}$ becomes extremal at the hypersurface described by (35). Therefore the correct equations of motion could also 
be obtained by varying $\mathcal{A}$ only on this hypersurface. Accordingly, it is allowed to eliminate $\Phi$ in favour of the vector potential $\vec{A}$ by inserting Eq. (35) into $L[\dot{\vec{A}}, \vec{A}, \Phi, t]$ arriving at $L^{\prime}[\dot{\vec{A}}, \vec{A}, t]=L[\dot{\vec{A}}, \vec{A}, \Phi[\dot{\vec{A}}, \vec{A}, t], t]$.

The next important step is to expand the remaining fields into an appropriate basis set exploiting the gauge condition. The irreducible degrees of freedom of $\vec{A}$ with $\nabla \vec{A}=0$ were described by an expansion into a complete set of real, orthonormal and transverse functions $\vec{f}_{a}(\vec{r})$ (see appendix):

$$
\vec{A}(\vec{r}, t)=q_{a}(t) \vec{f}_{a}(\vec{r}) \quad, \quad q_{a}(t)=\int d^{3} r \vec{A}(\vec{r}, t) \vec{f}_{a}(\vec{r}) \quad .
$$

Insertion of this expansion into (35) allows to express $\Phi$ completely in terms of the variables $q$ and $\dot{q}$ :

$$
\begin{aligned}
\Phi(\vec{r}, t) & =q_{a}(t) \varphi_{a}(\vec{r}, t)+\dot{q}_{a}(t) \chi_{a}(\vec{r}, t) \\
\varphi_{a}(\vec{r}, t) & =-\int d^{3} r^{\prime} G\left(\vec{r}, \vec{r}^{\prime}, t\right) \nabla^{\prime}\left(\left(\varepsilon\left(\vec{r}^{\prime}, t\right)-1\right) \vec{\beta}\left(\vec{r}^{\prime}, t\right) \times\left(\nabla^{\prime} \times \vec{f}_{a}\left(\vec{r}^{\prime}\right)\right)\right)+\mathcal{O}\left(\vec{\beta}^{2}\right),(38 \\
\chi_{a}(\vec{r}, t) & =-\int d^{3} r^{\prime} G\left(\vec{r}, \vec{r}^{\prime}, t\right) \nabla^{\prime}\left(\varepsilon\left(\vec{r}^{\prime}, t\right) \vec{f}_{a}\left(\vec{r}^{\prime}\right)\right)+\mathcal{O}\left(\vec{\beta}^{2}\right) .
\end{aligned}
$$

The scalar potential does no longer appear as a degree of freedom. We can express the physical fields:

$$
\begin{aligned}
& \vec{E}(\vec{r}, t)=\dot{q}_{a}(t) \vec{f}_{a}(\vec{r})+q_{a}(t) \nabla \varphi_{a}(\vec{r}, t)+\dot{q}_{a}(t) \nabla \chi_{a}(\vec{r}, t) \\
& \vec{B}(\vec{r}, t)=-q_{a}(t) \nabla \times \vec{f}_{a}(\vec{r}) .
\end{aligned}
$$

Insertion of these decompositions into the Lagrangian Eq. (23) leads to

$$
\begin{aligned}
L(q, \dot{q}, t) & =\int d^{3} r \mathcal{L}=\int d^{3} r\left(\mathcal{L}_{0}+\mathcal{L}_{1}(t)\right) \\
& =\frac{1}{2}\left(\varepsilon_{\infty} \dot{q}_{a}^{2}-\Omega_{a}^{2} q_{a}^{2}\right)+q_{a} \mathcal{X}_{a b}(t) q_{b}+q_{a} \mathcal{Y}_{a b}(t) \dot{q}_{b}+\dot{q}_{a} \mathcal{Z}_{a b}(t) \dot{q}_{b}
\end{aligned}
$$

Now we have a Lagrangian expressed by an independent set of variables $q$, that contains no constraints. The Euler-Lagrange equations

$$
\frac{d}{d t} \frac{\partial L}{\partial \dot{q}_{a}}=\frac{\partial L}{\partial q_{a}}
$$

lead to the Maxwell equation $\dot{\vec{D}}=\nabla \times \vec{H}$ after multiplication with $\vec{f}_{a}(\vec{r})$ and reidentification of the fields. The other equations - especially $\nabla \vec{D}=0$ - are automatically satisfied. Now we can derive the canonical momenta $p$ and their field representations $\vec{\Pi}$ (see appendix):

$$
\vec{\Pi}=p_{a} \vec{f}_{a}=\frac{\partial L}{\partial \dot{q}_{a}} \vec{f}_{a}=\left(\varepsilon \vec{E}+(\varepsilon-1) \vec{B} \times \vec{\beta}+\mathcal{O}\left(\vec{\beta}^{2}\right)\right)_{\perp}=\vec{D}
$$


It turns out that the canonical momentum is equal to $\vec{D}$ also in this general case. This is not obvious because the terms including $\Phi$ contain also $\dot{q}$; but performing the calculations we see that the contribution from $\Phi(q, \dot{q}, t)$ to $p$ vanishes due to $\nabla \vec{D}=0$ (see appendix):

$$
\frac{\delta}{\delta \dot{\overrightarrow{A_{\perp}}}} L[\dot{\vec{A}}, \vec{A}, \Phi[\dot{\vec{A}}, \vec{A},], t] \equiv \vec{f}_{a} \frac{\partial}{\partial \dot{q}_{a}} L(q, \dot{q}, t)=\frac{\delta}{\delta \vec{E}} L[\vec{E}, \vec{B}, t]
$$

The Hamiltonian now becomes:

$$
H(p, q, t)=p_{a} \dot{q}_{a}-L=\int d^{3} r \mathcal{H}(p, q, t)
$$

with

$$
\mathcal{H}(p, q, t)=\frac{1}{2}\left(\frac{1}{\varepsilon(\vec{r}, t)} \vec{\Pi}^{2}+(\nabla \times \vec{A})^{2}\right)+\frac{\varepsilon(\vec{r}, t)-1}{\varepsilon(\vec{r}, t)} \vec{\beta}(\vec{r}, t)(\vec{\Pi} \times(\nabla \times \vec{A}))+\mathcal{O}\left(\vec{\beta}^{2}\right)
$$

respectively

$$
\mathcal{H}(p, q, t)=\frac{1}{2}(\vec{E} \vec{D}+\vec{B} \vec{H})
$$

We have thus derived the Hamiltonian which indeed has the same form as the one obtained in the naive way (32), except for the term $\vec{\Pi} \nabla \Phi$. Now $\Phi$ and $\vec{A}_{\|}$are no longer degrees of freedom and the gauge condition is automatically satisfied. As a consequence the term $\vec{\Pi} \nabla \Phi$ disappears.

\subsection{Quantization}

Based on the Lagrangian $L(\dot{q}, q, t)$ obtained above it is possible to carry out the procedure of the canonical quantization via defining the following commutation relations:

$$
\left[\hat{q}_{a}(t), \hat{q}_{b}(t)\right]=\left[\hat{p}_{a}(t), \hat{p}_{b}(t)\right]=0 \quad, \quad\left[\hat{q}_{a}(t), \hat{p}_{b}(t)\right]=i \delta_{a b}
$$

Multiplication with the eigenfunctions $\left\{f_{a}\right\}$ leads to the commutation relations for the fields:

$$
\left[\hat{A}^{i}(\vec{r}, t), \hat{A}^{j}\left(\vec{r}^{\prime}, t\right)\right]=\left[\hat{\Pi}^{i}(\vec{r}, t), \hat{\Pi}^{j}\left(\vec{r}^{\prime}, t\right)\right]=0
$$

and

$$
\left[\hat{A}^{i}(\vec{r}, t), \hat{\Pi}^{j}\left(\vec{r}^{\prime}, t\right)\right]=i \delta_{\perp}^{i j}\left(\vec{r}-\vec{r}^{\prime}\right)
$$


Eq. (51) can be used to obtain a medium-independent (i.e. for arbitary $\varepsilon(\vec{r}, t)$ and $\vec{\beta}(\vec{r}, t)$ ) commutation relation between the fields $\vec{B}=-\nabla \times \vec{A}$ and $\vec{D}=\vec{\Pi}$ (see e.g. [11) :

$$
\left[\hat{B}^{i}(\vec{r}, t), \hat{D}^{j}\left(\vec{r}^{\prime}, t\right)\right]=i \epsilon^{i j k} \partial_{k} \delta\left(\vec{r}-\vec{r}^{\prime}\right)
$$

In the quantization prescription based on the decomposition presented above the Coulomb gauge condition (and also $\nabla \vec{D}=0$ ) appears as an operator identity. This leads to

$$
\forall|\psi\rangle, n: \quad\left\langle\psi\left|(\nabla \hat{\vec{A}})^{n}\right| \psi\right\rangle=0
$$

i.e., the fixing of the gauge removes all longitudinal photons. But for the scalar photons this is not the case for any variation of $\varepsilon(\vec{r}, t)$ or $\vec{\beta}(\vec{r}, t)$ :

$$
\left\langle 0\left|\hat{\Phi}^{2}(\vec{r}, t)\right| 0\right\rangle \neq 0
$$

\subsection{Interaction Hamiltonian}

Now we are in the position to apply the formalism presented in section 2. After separating the undisturbed $\hat{H}_{0}$, the interaction Hamiltonian can be cast into the form:

$$
\hat{\mathcal{H}}_{1}=\frac{1}{2}\left(\frac{1}{\varepsilon(\vec{r}, t)}-\frac{1}{\varepsilon_{\infty}}\right) \hat{\vec{\Pi}}^{2}+\frac{\varepsilon(\vec{r}, t)-1}{\varepsilon(\vec{r}, t)} \vec{\beta}(\vec{r}, t)(\hat{\vec{\Pi}} \times(\nabla \times \hat{\vec{A}}))+\mathcal{O}\left(\vec{\beta}^{2}\right)
$$

There are two terms giving rise to disturbances of the vacuum and thus contributing to the production of photons: the first term due to the change of the dielectric function $\varepsilon(\vec{r}, t)-$ the squeezing term - and the second one related to $\vec{\beta}(\vec{r}, t)$ - the velocity term.

The assumption of localized perturbations as stated in section 2 requires $\varepsilon(\vec{r}, t) \rightarrow \varepsilon_{\infty}$ and $\vec{\beta}(\vec{r}, t) \rightarrow 0$ for $|\vec{r}| \rightarrow \infty$ and for $t \rightarrow \pm \infty$.

\section{Squeezing effect}

As indicated in section 2, the general form for the particle production due to the disturbance $\hat{\mathcal{H}}_{1}$ turns out to be:

$$
\begin{aligned}
\left\langle\hat{N}_{\vec{k} \lambda}\right\rangle & =\left\langle\psi(t \rightarrow \infty)\left|\hat{N}_{\vec{k} \lambda}\right| \psi(t \rightarrow \infty)\right\rangle \\
& =\int d^{4} x \int d^{4} x^{\prime}\left\langle 0\left|\hat{\mathcal{H}}_{1}(\underline{x}) \hat{N}_{\vec{k} \lambda} \hat{\mathcal{H}}_{1}\left(\underline{x}^{\prime}\right)\right| 0\right\rangle+\mathcal{O}\left(\hat{H}_{1}^{3}\right)=N_{\vec{k} \lambda}+\mathcal{O}\left(\hat{H}_{1}^{3}\right)
\end{aligned}
$$

Now we shall evaluate this expression for $\vec{\beta}=\overrightarrow{0}$, neglecting the effects of any matter flow, i.e., for the pure squeezing contribution:

$$
\hat{\mathcal{H}}_{1}(\vec{r}, t)=\frac{1}{2}\left(\frac{1}{\varepsilon(\vec{r}, t)}-\frac{1}{\varepsilon_{\infty}}\right) \hat{\vec{\Pi}}^{2}(\vec{r}, t)=\xi(\vec{r}, t) \hat{\vec{\Pi}}^{2}(\vec{r}, t)
$$


The mode expansion in the $\hat{H}_{0}$-dynamics leads to:

$$
\hat{\vec{\Pi}}=\frac{i}{\sqrt{V}} \underset{\vec{k} \lambda}{f_{f}} \sqrt{\frac{\omega_{\vec{k}}}{2}}\left(\hat{a}_{\vec{k} \lambda}^{+} \vec{e}_{\vec{k} \lambda} e^{i \underline{k} \underline{x}}-\text { h.c. }\right)
$$

with $\underline{k}=\left(\vec{k}, \omega_{\vec{k}}\right)=\left(\vec{k}, k / \sqrt{\varepsilon_{\infty}}\right)$ and $\vec{k} \vec{e}_{\vec{k} \lambda}=0$. Inserting this expansion into $\hat{H}_{1}$ we arrive at :

$$
\begin{aligned}
& N_{\vec{k} \lambda}=\int d^{4} x \int d^{4} x^{\prime}\langle 0| \xi(\underline{x}) \underset{\vec{k}_{1} \lambda_{1}}{£} \underset{\vec{k}_{2} \lambda_{2}}{£} \frac{\sqrt{\omega_{\vec{k}_{1}} \omega_{\vec{k}_{2}}}}{2 V} \hat{a}_{\vec{k}_{1} \lambda_{1}} \vec{e}_{\vec{k}_{1} \lambda_{1}} \hat{a}_{\vec{k}_{2} \lambda_{2}} \vec{e}_{\vec{k}_{2} \lambda_{2}} e^{-i\left(\underline{k}_{1}+\underline{k}_{2}\right) \underline{x}} \\
& \times \hat{N}_{\vec{k} \lambda} \xi\left(\underline{x}^{\prime}\right) \underset{\vec{k}_{3} \lambda_{3}}{£} \underset{\vec{k}_{4} \lambda_{4}}{£} \frac{\sqrt{\omega_{\vec{k}_{3}} \omega_{\vec{k}_{4}}}}{2 V} \hat{a}_{\vec{k}_{3} \lambda_{3}}^{+} \vec{e}_{\vec{k}_{3} \lambda_{3}} \hat{a}_{\vec{k}_{4} \lambda_{4}}^{+} \vec{e}_{\vec{k}_{4} \lambda_{4}} e^{i\left(\underline{k}_{3}+\underline{k}_{4}\right) \underline{x}^{\prime}}|0\rangle .
\end{aligned}
$$

Performing all $\vec{k} \lambda$-summations we would obtain a distribution-like response function that is related to the 6-point correlation function. Here we proceed in a different way and carry out the $d^{4} x$-integration which yields a more compact form of the equation in terms of Fourier transformation $\mathcal{F}$ :

$$
\mathcal{F}: f(\underline{x}) \rightarrow \tilde{f}(\underline{k})=\int d^{4} x f(\underline{x}) e^{i \underline{k} \underline{x}} .
$$

Accordingly, we obtain

$$
\begin{aligned}
& N_{\vec{k} \lambda}=\underset{\vec{k}_{1} \lambda_{1}}{£} \underset{\vec{k}_{2} \lambda_{2}}{£} \underset{\vec{k}_{3} \lambda_{3}}{\&} \underset{\vec{k}_{4} \lambda_{4}}{\&} \frac{\sqrt{\omega_{\vec{k}_{1}} \omega_{\vec{k}_{2}} \omega_{\vec{k}_{3}} \omega_{\vec{k}_{4}}}}{4 V^{2}} \tilde{\xi}^{*}\left(\underline{k}_{1}+\underline{k}_{2}\right) \widetilde{\xi}\left(\underline{k}_{3}+\underline{k}_{4}\right) \times \\
& \left(\vec{e}_{\vec{k}_{1} \lambda_{1}} \vec{e}_{\vec{k}_{2} \lambda_{2}}\right)\left(\vec{e}_{\vec{k}_{3} \lambda_{3}} \vec{e}_{\vec{k}_{4} \lambda_{4}}\right)\left\langle 0\left|\hat{a}_{\vec{k}_{1} \lambda_{1}} \hat{a}_{\vec{k}_{2} \lambda_{2}} \hat{N}_{\vec{k} \lambda} \hat{a}_{\vec{k}_{3} \lambda_{3}}^{+} \hat{a}_{\vec{k}_{4} \lambda_{4}}^{+}\right| 0\right\rangle
\end{aligned}
$$

and evaluate the expectation values:

$$
N_{\vec{k} \lambda}=\varlimsup_{\overrightarrow{k^{\prime}} \lambda^{\prime}} \frac{\omega_{\vec{k}} \omega_{\vec{k}^{\prime}}}{V^{2}}\left|\widetilde{\xi}\left(\underline{k}+\underline{k^{\prime}}\right)\right|^{2}\left(\vec{e}_{\vec{k} \lambda} \vec{e}_{\vec{k}^{\prime} \lambda^{\prime}}\right)^{2} .
$$

Requiring again $\tilde{\xi}(\underline{k})$ to be finite for $\vec{k} \rightarrow \overrightarrow{0}$ and integrable (see section 2) power counting reveals that the number of particles per mode possess a leading term $N_{\vec{k} \lambda} \sim \omega_{\vec{k}}$ at low energies leading to a non-thermal spectrum $e(\omega)=\mathcal{O}\left(\omega^{4}\right)$ also in this case. 
With the aid of the completeness relation for the polarisation vectors $\vec{e}_{\vec{k} \lambda}$ and $\vec{e}_{\vec{k}}=\vec{k} / k$ :

$$
\vec{e}_{\vec{k}} \otimes \vec{e}_{\vec{k}}+\sum_{\lambda} \vec{e}_{\vec{k} \lambda} \otimes \vec{e}_{\vec{k} \lambda}=\mathbb{1}
$$

we arrive at

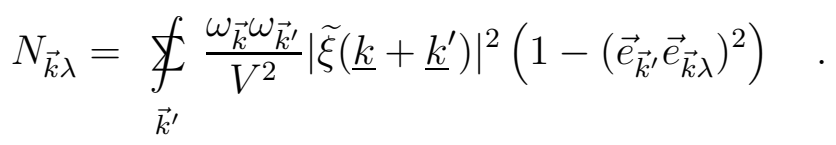

For reasons of simplicity we consider radialsymmetric functions $\xi(\vec{r}, t)=\xi(r, t)$ and expand the Fourier transform $\widetilde{\xi}$ into a Taylor-series:

$$
\widetilde{\xi}(\underline{k})=\int d t e^{i \omega t} \int d^{3} r e^{i \vec{k} \vec{r}} \xi(r, t)=\int d t e^{i \omega t} \sum_{n=0}^{\infty} \mathcal{M}_{n}(t)\left(\vec{k}^{2}\right)^{n}=\sum_{n=0}^{\infty} \widetilde{\mathcal{M}}_{n}(\omega)\left(\vec{k}^{2}\right)^{n}
$$

assuming that the $\mathcal{M}_{n}(t)$ which are related to the Mellin transform of $\xi(r, t)$ exist, i.e. that $\xi(r, t)$ is sufficiently localized.

The zero term is proportional to the volume $\mathcal{V}$ of the disturbance. For example for a bubble described by $\varepsilon(\vec{r}, t)=\varepsilon_{\infty}+\left(1-\varepsilon_{\infty}\right) \Theta(R(t)-r)$ it follows: $\mathcal{M}_{0}(t)=\frac{1}{2}\left(1-\varepsilon_{\infty}^{-1}\right) \mathcal{V}(t)$.

Inserting Eq. (65) into Eq. (64) leads to:

$$
N_{\vec{k} \lambda}=\oiint_{\vec{k}^{\prime}} \frac{\omega_{\vec{k}} \omega_{\vec{k}^{\prime}}}{V^{2}}\left(1-\left(\vec{e}_{\vec{k}^{\prime}} \vec{e}_{\vec{k} \lambda}\right)^{2}\right) \sum_{n=0}^{\infty} \sum_{m=0}^{\infty} \widetilde{\mathcal{M}}_{n}^{*}\left(\omega_{\vec{k}}+\omega_{\vec{k}}^{\prime}\right) \widetilde{\mathcal{M}}_{m}\left(\omega_{\vec{k}}+\omega_{\vec{k}}^{\prime}\right)\left(\vec{k}+\vec{k}^{\prime}\right)^{2(n+m)} .
$$

For the radialsymmetric case $N_{\vec{k} \lambda}$ does not depend on $\lambda$ and $\vec{e}_{\vec{k}}$, therefore we turn to $N_{k}=$ $\sum_{\lambda} N_{\vec{k} \lambda}$ :

$$
N_{k}=\oiint_{\vec{k}^{\prime}} \frac{\omega_{\vec{k}} \omega_{\vec{k}^{\prime}}}{V^{2}}\left(1+\left(\vec{e}_{\vec{k}} \vec{e}_{\vec{k}^{\prime}}\right)^{2}\right) \sum_{n=0}^{\infty} \sum_{m=0}^{\infty} \widetilde{\mathcal{M}}_{n}^{*}\left(\omega_{\vec{k}}+\omega_{\vec{k}}^{\prime}\right) \widetilde{\mathcal{M}}_{m}\left(\omega_{\vec{k}}+\omega_{\vec{k}}^{\prime}\right)\left(\vec{k}+\vec{k}^{\prime}\right)^{2(n+m)}
$$

Evaluating the total radiated energy

$$
E=\oiint_{\vec{k}} \omega_{\vec{k}} N_{k} \rightarrow \frac{V}{(2 \pi)^{3}} \int d^{3} k \frac{k}{\sqrt{\varepsilon_{\infty}}} N_{k}
$$


we arrive after some calculations at

$$
E=\sum_{n=0}^{\infty} \sum_{m=0}^{\infty} \int d t\left(\left(\frac{d}{d t}\right)^{4+2 n} \mathcal{M}_{n}(t)\right) \mathcal{G}^{n m}\left(\left(\frac{d}{d t}\right)^{4+2 m} \mathcal{M}_{m}(t)\right)
$$

with a geometry-independent matrix $\mathcal{G}^{n m}=\mathcal{G}^{n m}\left(n+m, \varepsilon_{\infty}\right)$ (see appendix).

The lowest (volume) term becomes for the bubble example

$$
E^{00}=\mathcal{G}^{00} \int d t\left(\mathcal{M}_{0}^{(4)}(t)\right)^{2}=\left(\frac{\varepsilon_{\infty}}{2 \pi}\right)^{3} \frac{1}{3 \cdot 5 \cdot 7}\left(\frac{1-\varepsilon_{\infty}^{-1}}{2}\right)^{2} \int d t \dddot{\mathcal{V}}^{2}(t)
$$

If we assume that the dynamics of the disturbance can be described by a maximal characteristic length scale $R_{\max }$ and minimal time scale $T_{\min }$, this term is of order $\mathcal{O}\left(R_{\max }^{6} T_{\min }^{-7}\right)$. Further terms contain additional factors of order $\mathcal{O}\left(R_{\max }^{2} T_{\min }^{-2}\right)$ and can be neglected for the case $R_{\max } / T_{\min } \ll 1$.

\section{$5 \quad$ Velocity effect}

As one can see in section 2, the spectrum of the photons produced by the dynamical Casimir effect depends very strongly on the concrete form of the disturbance. Now we shall study some examples for the profile of the velocity $\vec{\beta}(\vec{r}, t)$ :

The first example corresponds to the radialsymmetric flow of an incompressible fluid around a bubble with the time dependent radius $R(t)$ :

$$
\vec{\beta}(\vec{r}, t)=\dot{R}(t) \vec{e}_{r} R^{2}(t) / r^{2}
$$

The Fourier transform $\widetilde{\vec{\beta}}\left(\vec{k}, \omega_{\vec{k}}\right)$ remains finite in the limit $\vec{k} \rightarrow \overrightarrow{0}$ and is of order $R_{\max }^{3}$. Therefore the spectrum of the created photons behaves as $\omega^{4}+\mathcal{O}\left(\omega^{5}\right)$ for smooth and localized functions $R(t)$ and the velocity contribution is for this case of the same order as the squeezing term. (The perturbation Hamiltonian for the squeezing effect is also of order $R_{\max }^{3}$ due to the $d^{3} r$ integration.) For the incompressible case the flow $\oint \vec{\beta} d \vec{A}$ of the fluid is instantaneously constant even for $|\vec{r}| \rightarrow \infty$. If we assume a more realistic situation, where $\vec{\beta}(\vec{r}, t)$ yields relevant contributions only over a bounded volume of order $R_{\max }^{3}$, the velocity effect turns out to be of order $R_{\max }^{4}$ and can therefore be neglected in those cases, where $R_{\max }$ is sufficiently small. (The $\mathcal{O}\left(R_{\max }^{4}\right)$-behaviour results from the $d^{3} r$-integration that implies $\mathcal{O}\left(R_{\text {max }}^{3}\right)$ and the assumption, that $\vec{\beta}(\vec{r}, t)$ is still of order $R_{\max }$.)

For the second example:

$$
\vec{\beta}(\vec{r}, t)=\dot{R}(t) \vec{e}_{r}
$$

that is studied in Ref. [10], one obtains a Fourier transform that is divergent for $\vec{k} \rightarrow \overrightarrow{0}$. This demonstrates the highly nonlocalized character of this profile which results in an increasing flow for $|\vec{r}| \rightarrow \infty$. Of course, for that case one could not provide the prediction $e(\omega) \sim \omega^{4}+\mathcal{O}\left(\omega^{5}\right)$. 
For a third example we study the situation of a rigidly moving medium with

$$
\varepsilon(\vec{r}, t)=\varepsilon_{\infty} \quad \text { and } \quad \vec{\beta}(\vec{r}, t)=\vec{\beta}(t) .
$$

For that case Eq. (34) for $\Phi$ is easyly solvable: $\Phi=\left(\varepsilon_{\infty}^{-1}-1\right) \vec{\beta} \vec{A}+\mathcal{O}\left(\vec{\beta}^{2}\right)$. Calculating the perturbation Hamiltonian $\hat{H}_{1}$ we find no photon production to first order in $\vec{\beta}$ (nonrelativistic limit): $\hat{H}_{1}|0\rangle=\mathcal{O}\left(\vec{\beta}^{2}\right)$. This implies that for the case of a purely time-varying velocity the effect will be of order $N_{\vec{k} \lambda}=\mathcal{O}\left(\vec{\beta}^{4}\right)$.

\section{Discussion}

\subsection{Finite temperatures}

The response theory approach to quantum radiation allows for the incorporation of finite temperature effects. Instead of using the time evolution for the pure quantum state Eq. (9) one has to consider the equation of motion for the statistical operator $\hat{\rho}$ :

$$
\frac{d \hat{\rho}}{d t}=-i\left[\hat{H}_{1}, \hat{\rho}\right]
$$

and Eq. (12) becomes

$$
\left\langle\hat{N}_{\vec{k} A}\right\rangle=\operatorname{Tr}\left\{\hat{\rho}_{0} \mathcal{T}\left[\exp \left(i \int d^{4} x \hat{\mathcal{H}}_{1}(\underline{x})\right)\right] \hat{N}_{\vec{k} A} \mathcal{T}\left[\exp \left(-i \int d^{4} x^{\prime} \hat{\mathcal{H}}_{1}\left(\underline{x}^{\prime}\right)\right)\right]\right\} .
$$

The zero-order term contains the undisturbed spectrum arising from $\hat{\rho}_{0}$. The contribution of first order in $\hat{H}_{1}$ vanishes, if we assume a $\hat{\rho}_{0}$ related to the thermodymamic equilibrium:

$$
\hat{\rho}_{0}=\hat{\rho}(t \rightarrow-\infty)=\exp \left(-\beta \hat{H}_{0}\right) / Z_{0} \quad .
$$

The terms of second order contain the production of particles and the interaction between the thermal radiation field and the disturbance.

\subsection{Permeable media}

The approach presented above is generalizable to the case of media described by $\varepsilon(\vec{r}, t), \mu(\vec{r}, t)$ and $\vec{\beta}(\vec{r}, t)$, if one adds a term proportional to $(\mu-1) u_{\mu} \stackrel{*}{F^{\mu}}{ }_{\nu}{\stackrel{*}{F^{\nu}}}_{\rho} u^{\rho}$ with the dual tensor $\stackrel{*}{F}_{\mu \nu}=\epsilon_{\mu \nu \rho \sigma} F^{\rho \sigma}$ to the Lagrangian.

For pure magnetic disturbances $(\varepsilon=1)$ the calculations proceed in the same way, if one replaces the potentials $\vec{A}$ and $\Phi$ by the dual potentials $\vec{\Lambda}$ and $\Gamma$ (see e.g. [17]), defined via:

$$
\vec{D}=\nabla \times \vec{\Lambda} \quad, \quad \vec{H}=\dot{\vec{\Lambda}}+\nabla \Gamma .
$$

A possible application could be the case of magnetic phase transitions, e.g. in connection to superconductivity. 


\subsection{Contribution to sonoluminescence}

There have been various attempts to interprete the phenomenon of sonoluminescence [22] as an effect of quantum radiation [23, 9, 10]. In order to estimate the contribution of QR it is important to determine the characteristic parameters (time- and lenght scales) involved in the dynamics of the bubble [24, 25]. Calculations of the stationary Casimir energy (see Refs. [26] and [27]) cannot account for the dynamical character of the effect. Without an upper limit for the volume $R_{\max }^{3}$ and the time interval $T_{\max }$ of the disturbance and a frequency cutoff $K_{c}$ the radiated energy could diverge due to the space-time integration and the mode summation, if the perturbation functions are not localized and smooth. But if we assume a localized disturbance and a frequency cutoff due to the fact, that $\varepsilon$ differs only from 1 for wave numbers $k<K_{c}$, an estimation of the upper limit for the order of magnitude is possible. We envisage Eq. (62) as an example for the general case of the quadratic response, assuming that the higher order terms are much smaller:

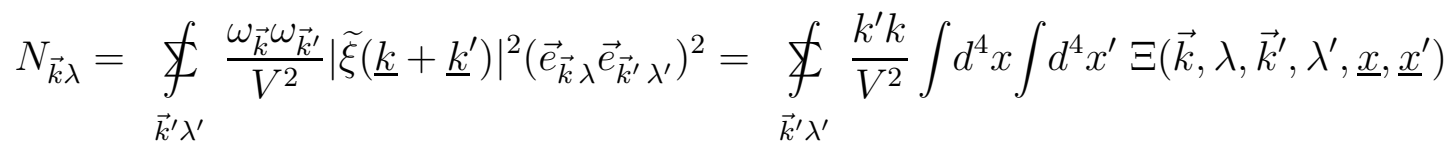

with a function $\Xi$ that is of order 1 . The order of magnitude of the space-time integrations and the mode sum can be estimated using $R_{\max }, T_{\max }, K_{c}$ and the quantization volume $V$ :

$$
\int d^{4} x \rightarrow \mathcal{O}\left(T_{\max } R_{\max }^{3}\right) \quad \text { and } \underset{\vec{k}}{\underset{f}{f}} \rightarrow \mathcal{O}\left(V K_{c}^{3}\right)
$$

This leads to $N_{\vec{k} \lambda}^{\max }=\mathcal{O}\left(T_{\max }^{2} R_{\max }^{6} K_{c}^{5} / V\right)$ which depends still on the quantization volume $V$. But in the total energy as a physical observable $V$ of course does not enter:

$$
E^{\max }=£ \omega_{\vec{k}} N_{\vec{k} \lambda}^{\max } \rightarrow \mathcal{O}\left(R_{\max }^{6} T_{\max }^{2} K_{c}^{9}\right)
$$

Another result of this approach is the fact that the Casimir contribution to sonoluninescence should display a $\omega^{4}$-spectrum for small $\omega$ if we assume that the suppositions of section 2 are fulfilled. This provides a possibility to distinguish this effect from others (e.g. photon emission due to heating the gas in the bubble) which behave like $\omega^{3}$. 


\section{Acknowledgement}

G. S. acknowledges support by BMBF, DFG and GSI.

\section{Appendix}

We have to introduce a complete set of real, orthonormal and transverse eigenfunctions $\vec{f}_{a}(\vec{r})$ of the Laplace operator:

$$
\begin{aligned}
& \nabla^{2} \vec{f}_{a}(\vec{r})=-\Omega_{a}^{2} \vec{f}_{a}(\vec{r}), \\
& \forall a \quad \nabla \vec{f}_{a}(\vec{r})=0, \\
& \int d^{3} r \vec{f}_{a}(\vec{r}) \vec{f}_{b}(\vec{r})=\delta(a, b), \\
& \& f_{a}^{i}(\vec{r}) f_{a}^{j}\left(\vec{r}^{\prime}\right)=\delta_{\perp}^{i j}\left(\vec{r}-\vec{r}^{\prime}\right) .
\end{aligned}
$$

$a$

Accordingly, the field modes also satisfy the relation:

$$
\int d^{3} r\left(\nabla \times \overrightarrow{f_{a}}(\vec{r})\right)\left(\nabla \times \overrightarrow{f_{b}}(\vec{r})\right)=\Omega_{a}^{2} \delta_{a b}
$$

Let us show how to calculate the canonical momenta $p$ as well as their field representation $\vec{\Pi}=p_{a} \overrightarrow{f_{a}}$ from the Lagrangian:

$$
\begin{aligned}
p_{a} & =\frac{\partial L}{\partial \dot{q}_{a}}=\int d^{3} r \frac{\partial \mathcal{L}}{\partial \dot{q}_{a}}=\int d^{3} r \frac{\partial \mathcal{L}}{\partial \vec{E}} \frac{\partial \vec{E}}{\partial \dot{q}_{a}} \\
& =\int d^{3} r \vec{D}(\vec{r})\left(\vec{f}_{a}(\vec{r})+\nabla \chi_{a}(\vec{r}, t)\right)=\int d^{3} r \vec{D}(\vec{r}) \vec{f}_{a}(\vec{r})
\end{aligned}
$$

For the same reason $\Phi$ yields no direct contribution to $\frac{\partial L}{\partial q_{a}}$, i.e., to $\nabla \times \vec{H}$.

$$
\begin{aligned}
\vec{\Pi}(\vec{r})=p_{a} \vec{f}_{a}(\vec{r}) & =\int d^{3} r^{\prime} \vec{f}_{a}(\vec{r})\left(\vec{f}_{a}\left(\vec{r}^{\prime}\right) \vec{D}\left(\vec{r}^{\prime}\right)\right) \\
& =\int d^{3} r^{\prime} \vec{e}_{i} \delta_{\perp}^{i j}\left(\vec{r}-\vec{r}^{\prime}\right) D_{j}\left(\vec{r}^{\prime}\right)=\vec{D}_{\perp}(\vec{r})=\vec{D}(\vec{r})
\end{aligned}
$$

The transverse part $\vec{F}_{\perp}(\vec{r}, t)$ of an arbitrary vector field $\vec{F}(\vec{r}, t)=\vec{F}_{\|}(\vec{r}, t)+\vec{F}_{\perp}(\vec{r}, t)$ is defined as

$$
\nabla^{2} \vec{F}_{\perp}(\vec{r})=-\nabla \times(\nabla \times \vec{F}(\vec{r}))
$$


As a complete and orthonormal set of real and transversal modes we use

$$
f_{\vec{k} \lambda+}(\vec{r})=\sqrt{\frac{2}{V}} \cos (\vec{k} \vec{r}) \vec{e}_{\vec{k} \lambda}
$$

and

$$
f_{\vec{k} \lambda-}(\vec{r})=\sqrt{\frac{2}{V}} \sin (\vec{k} \vec{r}) \vec{e}_{\vec{k} \lambda}
$$

with polarization vectors $\vec{e}_{\vec{k} \lambda}=\vec{e}_{-\vec{k} \lambda}$. The $q$ are related to the creation/annihilation operators used in Eq. (58), e.g. for $q_{\vec{k} \lambda+}$ it follows:

$$
\hat{q}_{\vec{k} \lambda+}=\frac{1}{2 \sqrt{\omega_{\vec{k}}}}\left(\hat{a}_{\vec{k} \lambda}^{+}+\hat{a}_{\vec{k} \lambda}+\hat{a}_{-\vec{k} \lambda}^{+}+\hat{a}_{-\vec{k} \lambda}\right) .
$$

To be complete we note here the explicit expression for the Matrix $\mathcal{G}^{n m}$ used in section 4:

$$
\begin{array}{r}
\mathcal{G}^{n m}=\frac{\varepsilon_{\infty}^{3+n+m}}{(2 \pi)^{3}(8+2 n+2 m) !} \sum_{\ell=0}^{n+m}\left(\begin{array}{c}
m+n \\
\ell
\end{array}\right) 2^{\ell}\left((-1)^{\ell}+1\right)\left(\frac{1}{\ell+1}+\frac{1}{\ell+3}\right) \times \\
\sum_{s=0}^{n+m-\ell}\left(\begin{array}{c}
m+n-\ell \\
s
\end{array}\right)(4+\ell+2 s) !(3-\ell-2 s+2 n+2 m) !
\end{array} .
$$




\section{References}

[1] H. B. G. Casimir, Proc. K. Ned. Akad. Wet. 51 , 793 (1948)

[2] S. K. Lamoreaux, Phys. Rev. Lett. 78, 5 (1997)

[3] G. Plunien, B. Müller, W. Greiner, Phys. Rep. 134, 87 (1986)

[4] A. A. Grib, S. G. Mamayev, V. M. Mostepaneko, "Vacuum Quantum Effects in Strong Fields", (Friedmann Laboratory Publishing, St. Petersburg, 1994)

[5] M. Bordag, "Quantum Field Theory Under the Influence of External Conditions" (Teubner, Stuttgart, 1996)

[6] N. D. Birrell, P. C. W. Davies, "Quantum Fields in Curved Space", (Cambrigde University Press, Cambrigde, 1982)

[7] R. Schützhold, G. Plunien, G. Soff, e-preprint: quant-ph/9709008 accepted for publication in Phys. Rev. A (1998)

[8] G. Barton, C. Eberlein, Ann. Phys. (N.Y.) 227, 222 (1993)

[9] C. Eberlein, Phys. Rev. Lett. 76, 3842 (1996)

[10] C. Eberlein, Phys. Rev. A 53, 2772 (1996)

[11] M. Born, L. Infeld, Proc. Roy. Soc. London, Ser. A 147, 522 (1934); 150, 141 (1935)

[12] J. M. Jauch, K. M. Watson, Phys. Rev. 74, 950 (1948)

[13] T. A. Kennedy, E.M. Wright, Phys. Rev. A 38, 212 (1988)

[14] B. J. Dalton, E. S. Guerra, P. L. Knight, Phys. Rev. A 54, 2292 (1996)

[15] H. T. Dung, L. Knöll, D.-G. Welsch, e-preprint: quant-ph/9711039

[16] L.-M. Duan, G.-C. Guo, Phys. Rev. A 56, 925 (1997)

[17] M. Hillery, L. D. Mlodinow, Phys. Rev. A 30, 1860 (1984)

[18] P. D. Drummond, Phys. Rev. A 42, 6845 (1990)

[19] R. J. Glauber, M. Lewenstein, Phys. Rev. A 43, 467 (1991) 
[20] M. Bordag, K. Kirsten, D. V. Vassilevich, e-preprint: hep-th/9709084

[21] M. Henneaux, C. Teitelboim, "Quantization of Gauge Systems" (Princeton University Press, Princeton, New Jersey, 1992)

[22] B. P. Barber, R. A. Hiller, R. Löfstedt, S. J. Putterman, K. R. Weninger, Phys. Rep. 281, $65(1997)$

[23] J. Schwinger, Proc. Natl. Acad. Sci. USA 89, 4091 (1992); 89, 11118 (1992);

90, 958 (1993); 90, 2105 (1993); 90, 4505 (1993); 90, 7285 (1993); 91, 6473 (1994);

[24] B. Gompf, R. Günther, G. Nick, R. Pecha, W. Eisenmenger, Phys. Rev. Lett. 79, 1405 (1997)

[25] C. Slotta, U. Heinz, e-preprint: quant-ph/9711058

[26] C. E. Carlson, C. Molina-Páris, J. Pérez-Mercader, M. Visser e-preprint: hep-th/9609195

[27] K. A. Milton, Y. J. Ng, e-preprint: hep-th/9607186; e-preprint: hep-th/9707122 